\title{
LARGE SCALE STAR FORMATION: DENSITY WAVES, SUPERASSOCIATIONS AND PROPAGATION
}

\author{
Bruce G. Elmegreen \\ IBM Thomas J. Watson Research Center \\ P.O. Box 218, Yorktown Heights, N.Y. 10598 USA
}

\begin{abstract}
The hypothesis that density waves trigger star formation is critically examined. Much of the former evidence in favor of the hypothesis is shown to be inconsistent with modern observations. A comparison between galaxies with and without density waves reveals no significant difference in their star formation rates. A new role for density waves in the context of star formation might be based on four principles: 1. density waves are intrinsically strong, 2. the gas is compressed more than the stars in the wave, 3. star formation follows the gas, with no preferential trigger related to the wave itself, and 4. regions of star formation are larger in the spiral arms than they are between the arms. This new role for density waves is primarily one of organization: the waves place most of the gas in the arms, so most of the star formation is in the arms too. The waves also promote the coagulation of small clouds into large cloud complexes, or superclouds, by what appears to be a combination of collisional agglomeration and large-scale gravitational instabilities. Special regions where density waves do trigger a true excess of star formation are discussed, and possible reasons for the difference between these triggering waves and the more common, organizing, waves are mentioned. Other aspects of large-scale star formation, such as the occurrence of kiloparsec-size regions of activity and kiloparsec-range propagation, are illustrated with numerous examples. The importance of these largest scales to the overall mechanism of star formation in galaxies is emphasized.
\end{abstract}

\section{INTRODUCTION}

Three aspects of star formation on a galactic scale are addressed here: 1. the relationship between galactic spiral arms and star formation; 2. the apparent coherence of some star formation on a kiloparsec scale, and 3. the propagation of star formation over kiloparsec distances.

The correspondence between star formation and galactic spiral arms was first noted for M31 by Baade and Mayall (1951), and then used by Morgan et al. $(1952,1953)$ to delineate the local spiral arms. Baade (1963) later commented that HII regions in galaxies often line up "like pearls on a necklace." One explanation given for this phenomenon is that spiral arms are density waves (Lin and Shu 1964) and density waves trigger star formation (Roberts 1969). An alternative explanation is that swing amplification in galactic disks triggers the formation of both stellar and gaseous arms, and that the compression and shocks which develop in the gaseous arms lead to star formation (Goldreich and Lynden-Bell 1965; Lynden-Bell 1966; Toomre 1981). The most recent interpretation is that propagating star 
formation produces all aspects of spiral arms (Gerola and Seiden 1978). The evidence for and against density-wave triggered star formation is summarized in $\S 2$. Although density waves exist in some galaxies, the current evidence favors no significant density-wave trigger for star formation, except in rare circumstances. The other two interpretations for spiral-arm star formation are discussed in $\S \S 2.4,2.5$ and 4 , in the context of points 2 and 3 , respectively.

Observations of star formation on a kiloparsec scale are reviewed in $\S 3$. Most star formation in spiral or irregular galaxies appears to operate coherently on scales of several hundred parsecs to a kiloparsec. Hubble (1936), Humason, Mayall and Sandage (1956), Wray and de Vaucouleurs (1980) and others attempted to use the largest of these giant regions as extragalactic distance calibrators. Possible origins for large-scale star formation are reviewed in $\S \S 2.5$ and 4.

Long-range propagating star formation is discussed in $\S 4$. The recognition of such propagation dates back to a comment by Baade (1963), that star formation in the galaxy IC1613 appears to "spread like a disease." A detailed study of Constellation III in the Large Magellanic Cloud (Westerlund and Mathewson 1966) illustrated how this spreading occurs. A few more examples are known today. Propagation appears to be an important component of any general theory of large-scale star formation because it introduces non-linear interactions between cloud formation, star formation and cloud disruption. Such interactions can produce vigorous star formation in non-equilibrium systems, which may include star-burst galaxies and extragalactic HII regions, yet control the equilibrium between cloud formation and cloud disruption in normal galaxies, thereby producing a steady star formation rate there.

\section{DO DENSITY WAVES TRIGGER STAR FORMATION?}

\subsection{Classical Evidence for Density-Wave-Triggered Star Formation}

One of the fundamental implications of the original density wave theory (Lin and Shu 1964) was that density waves trigger star formation. Optical photographs of grand design galaxies show spiral arms with much larger amplitudes than expected for pure density waves, so the wave pattern was thought to be amplified by star formation. Color photographs and surface photometry (Schweizer 1976; Talbot, Jensen and Dufour 1979; Wevers 1984) show directly that star formation is enhanced in spiral arms because the arms are bluer than the interarm regions. Age gradients through spiral arms, as in M33 (Dixon 1971; Courtès and DuboutCrillon 1971; Dubout-Crillon 1977; Humphreys and Sandage 1980) and M31 (Efremov 1970, 1980a, 1985; Efremov and Ivanov 1982), are consistent with this triggering hypothesis, as is the evidence for systematic streaming motions at the time of birth for local stars or clouds (Dixon 1968; Grosbø1 1977; Bash et al. 1977, 1981; Hilton and Bash 1982; Anderson, Hodge and Kennicutt 1983). Observations of a spiral arm/interarm brightness contrast in molecules that exceeds the brightness contrast in atoms (Cohen et al. 1980) suggests similarly that density waves trigger molecular cloud formation.

Less direct evidence for a density wave trigger came from the Schmidt (1959) "law" for star formation, which has a star formation rate proportional to the square of the local gas density, and from the observation of shocks in density waves (Mathewson, van der Kruit and Brouw 1972; Ondrechen 1985) as predicted from gas flow calculations (Roberts 1969; Pikel'ner 1970; Roberts and Yuan 1970). The Schmidt law suggested that the star formation rate per unit gas mass should be enhanced in spiral arms because of the generally larger gas density there. The presence of shocks allowed star formation to be triggered by a variety of methods: the compression of individual clouds (Roberts 1969; Jura 1976; Woodward 1976), a phase transition (Shu et al. 1972; Biermann et al. 1972), large-scale magnetic or 
gravitational instabilities (Mouschovias, Shu and Woodward 1974; Elmegreen 1979, 1982a; Cowie 1981; Balbus and Cowie 1985; Tomisaka 1985), or cloud-cloud collisions in a shock-type front (Casoli and Combes 1982; Kwan and Valdes 1983; Roberts and Hausman 1984; Hausman and Roberts 1984; Combes and Gerin 1985; Tomisaka 1984, 1985; Fukunaga and Tosa 1987).

The triggering hypothesis also explained the Luminosity Classification System for galaxies (van den Bergh 1960), which was supposed to be a sequence of varying shock strength (Roberts, Roberts and Shu 1975). Radial metallicity gradients (Jensen, Strom and Strom 1976) and star formation rate gradients (Talbot 1980) could be explained as well, because the star formation rate was supposed to be larger in the inner regions where the frequency of interactions between spiral arms and interstellar gas is larger.

All of these observations are consistent with the original concept of gas compression and star formation in weak density-wave spiral arms. New observations are beginning to change this concept, however. These changes are so fundamental that they force us to reconsider completely all of the possible interactions between density waves, interstellar matter and star formation. The full extent of the required change is not known yet, but the new concept that is emerging differs from the old in significant, and sometimes subtle, ways.

\subsection{Modern Evidence that Density Waves Seldom Trigger Star Formation}

The density-wave triggering hypothesis was proposed at a time when one of the most important problems in galactic structure was to find an explanation for the occurrence of long and symmetric spiral arms (Oort 1962; Lin and Shu 1967; Lin 1968). Relatively little attention was given to galaxies without such arms, although observations of these other galaxies were occasionally discussed (Sandage 1961; Woltjer 1965). Apparently no one compared the colors or star formation rates in the two types of galaxies to see if galaxies with strong wave-like spirals have more star formation than galaxies without strong waves. Such a comparison, discussed below, indicates that the average star formation rates in the two galaxy types are essentially equal.

Star formation appears to operate equally well with or without a density wave. Figure 1 shows two galaxies, NGC 4321 (M100) and NGC 5055 (M63), which have similar Hubble types $(\mathrm{Sbc})$, colors $\left([\mathrm{B}-\mathrm{V}]_{T}^{\circ}=0.67\right.$ and $0.64,[\mathrm{U}-\mathrm{B}]_{e}^{\prime}=0.1$ and 0.15 , respectively, from de Vaucouleurs et al. 1976) and CO emission line intensities (Chance et al. 1986), although their optical sizes differ $\left(R_{25}=21 \mathrm{kpc}\right.$ and $13 \mathrm{kpc}$, respectively, for a Hubble constant of $\left.75 \mathrm{~km} \mathrm{~s}^{-1} \mathrm{Mpc}^{-1}\right)$. The figures on the top are in the blue passband (4360 $\AA$ ) and on the bottom in the near-infrared $(8250 \AA$ from Elmegreen 1981$)$. The near-infrared passband shows the distribution of old stars in the disk, and reveals a density wave if one is present. The most obvious difference between the two galaxies is that NGC 4321 has a density wave that organizes the star formation into spiral arms, and NGC 5055 does not. One also gets the impression that the largest HII regions in NGC 4321 are larger than the largest in NGC 5055.

To assess the density wave content of galaxies in a systematic way, a large number ( $\sim 600)$ of galaxies were classified according to the morphology of their spiral arms (D.Elmegreen and B.Elmegreen 1986). A comparison between this classification system (Elmegreen and Elmegreen 1982) and the blue and near-infrared properties of galaxies (Elmegreen and Elmegreen 1984) suggests that the morphology of spiral arms is a reasonably good indicator of the presence of density waves. Spiral arm class 1 galaxies are very irregular (of the Magellanic type), arm classes 2, 3 and 4 are flocculent to various degrees, and arm classes 5 through 12 are grand design to various degrees, ranging from galaxies with patchy and poorly defined arms (M33; arm class 5), to galaxies with multiple arms (M101; arm class 9), to galaxies with long and symmetric arms (M100; arm class 12). Flocculent galaxies (arm classes 1-4) generally have no density waves, and grand design galaxies (arm classes 5-12) 

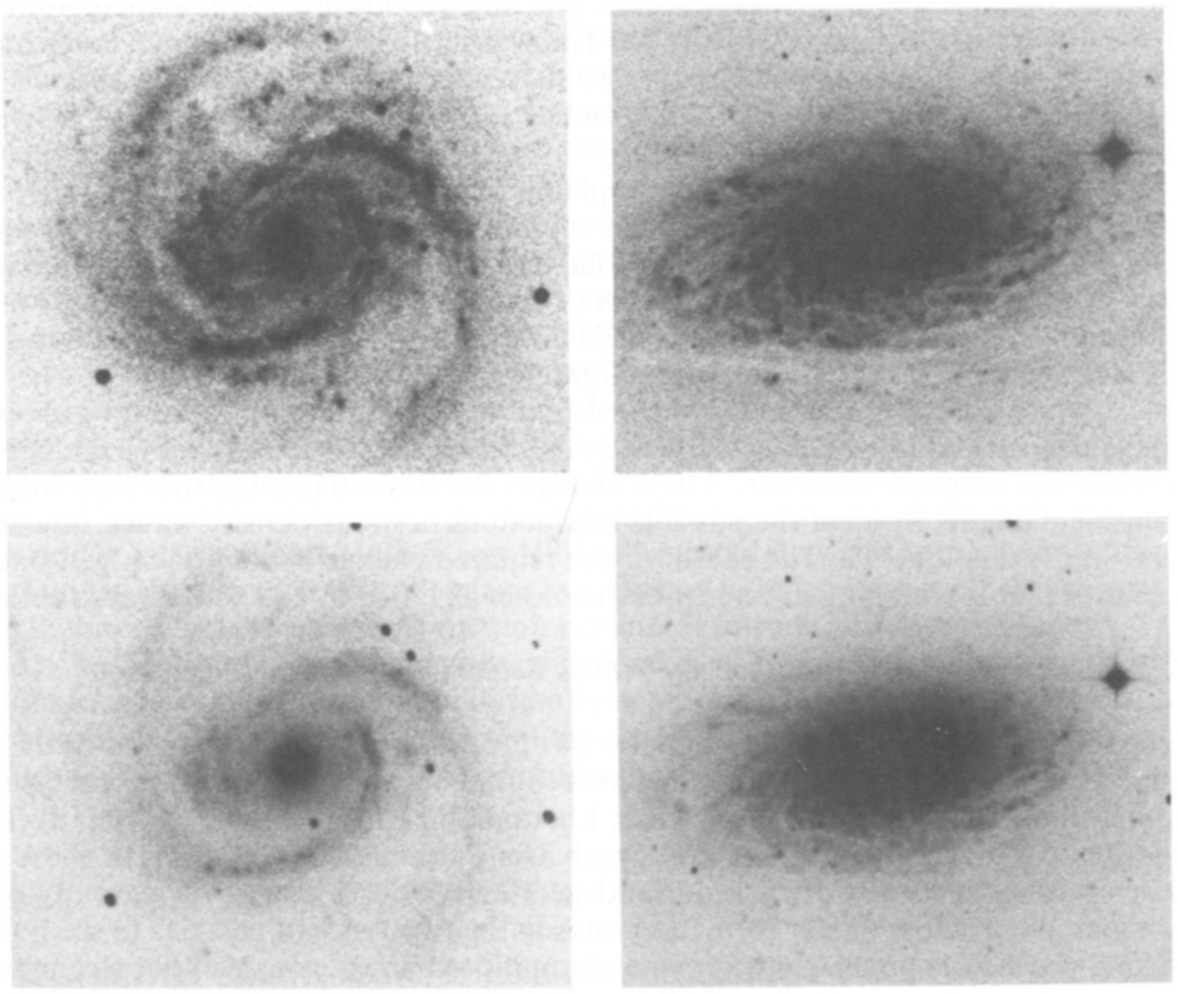

Figure 1: Blue (top) and near-inf rared (bottom) images of NGC 4321 (left) and NGC 5055 (right). The colors and Hubble types are similar for these two galaxies, but a density wave has organized the stars and gas into a grand design spiral pattern in NGC 4321 but not in NGC 5055.

generally have such waves, although there may be exceptions in each case.

We have searched without success (B.Elmegreen and D.Elmegreen 1986; hereafter EE86) for a correlation between the spiral arm class of a galaxy and the corrected B-V colors, surface brightnesses (from de Vaucouleurs et al. 1976) and star formation rates per unit area (from Kennicutt 1983 or Donas and Deharveng 1984). Figure 2a shows the B-V colors, and Figure $2 b$ the star formation rates per unit area (as available from Kennicutt 1983), plotted against the spiral arm classes of all of the face-on $\left(i<55^{\circ}\right)$ spiral galaxies in the following catalogues: Sandage (1961), Sandage and Tammann (1981), Sandage, Binggeli and Tammann (1985), Turner (1976), Turner and Gott (1976), Huchra and Geller (1982), and Geller and Huchra (1983). Different symbols represent different Hubble types: squares for Hubble types $\mathrm{Sa}$ to $\mathrm{Sb}$, circles for types Sbc to Scd, and diamonds for types $\mathrm{Sd}$ to $\mathrm{Sm}$. Within each arm class, the well-known variation of the color and star formation rate with Hubble type is obvious (squares have larger B-V values and smaller star formation rates than circles, which have larger B-V values and smaller star formation rates than diamonds). No significant variations of color or star formation rate per unit area occur for the different arm classes. This is also true for the star formation rates in Donas and Deharveng (1984), which are based on uv fluxes (EE86). The correlation found by Romanishin (1985), that grand design galaxies are slightly bluer than flocculent galaxies, was confirmed by us for the same sample of galaxies, but this correlation seems to disappear for the larger sample of galaxies 


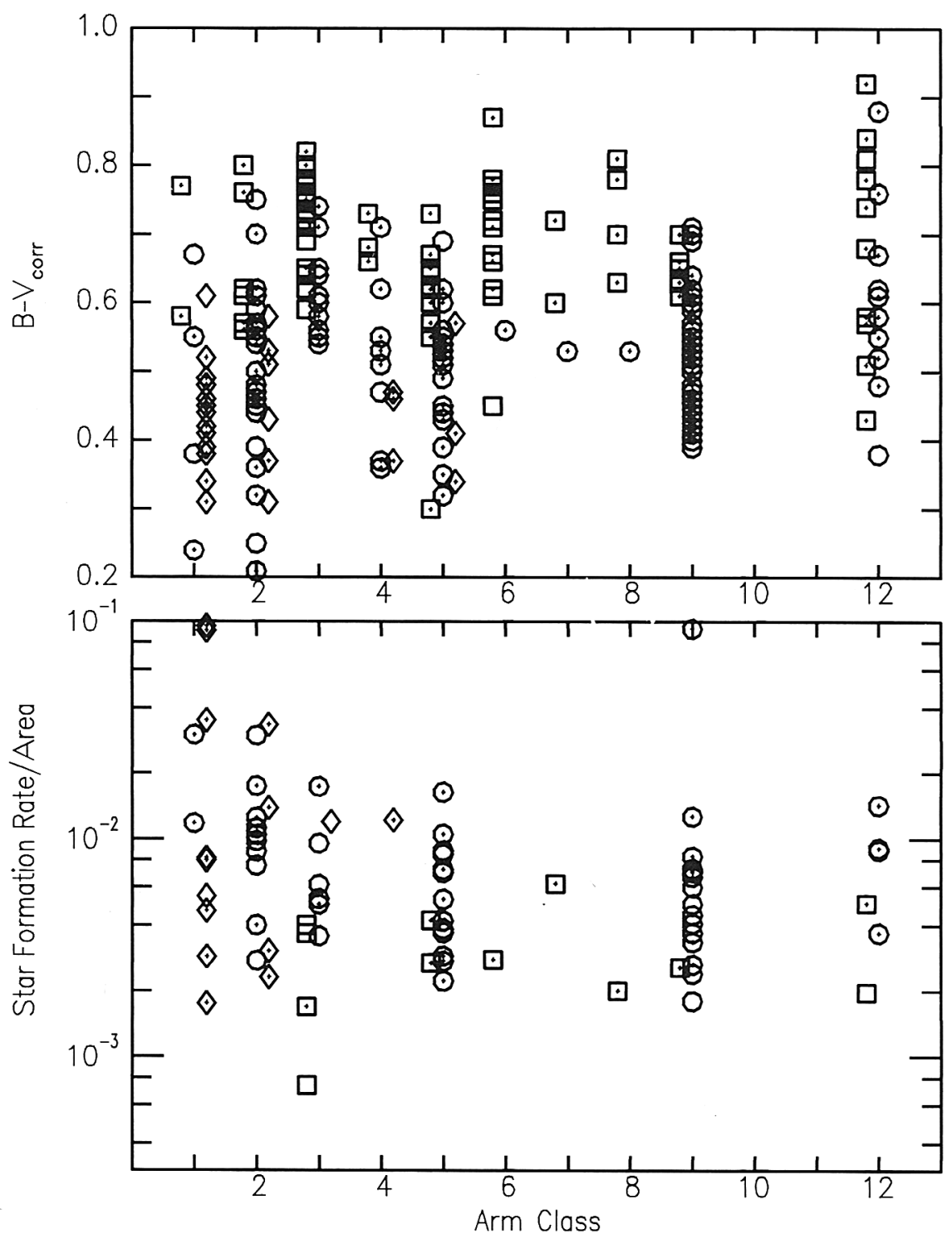

Figure 2: Galactic color corrected for inclination and extinction (figure a, top), and star formation rate per unit area, in $\mathbf{M}_{\odot} \mathbf{k p c}^{-2}$ (figure $b$, bottom), are shown as functions of arm class for face-on spiral galaxies. Symbols denote Hubble type: squares $=\mathrm{Sa}$ to $\mathrm{Sb}$, circles $=\mathrm{Sbc}$ to $\mathrm{Scd}$, diamonds $=\mathrm{Sd}$ to Sm. Symbols with dots are Hubble types SAB and SB, and without dots are types SA.

presented here. Galaxies with different arm classes were also found to have approximately the same average surface brightnesses (EE86). The slightly larger surface brightnesses for flocculent galaxies found by Phillips and Disney (1985) were not found by us. 
There is also no significant difference in color, star formation rate per unit area or surface brightness for galaxies in loose and dense groups (EE86). Galaxies in loose groups tend to be flocculent and galaxies in dense groups tend to be grand design (Elmegreen and Elmegreen 1983a). Thus, even if density waves are transient, the galaxies which spend most of their time with a wave (i.e., those in dense groups) appear to have no larger star formation rates per unit area than the galaxies which spend most of their time without a wave.

\subsection{New Interpretations for the Classical Evidence of Density-Wave Triggering}

Each observation formerly supporting the density-wave triggering hypothesis ( $\$ 2.1)$ has another interpretation that follows four basic points: 1 . density waves are strong; 2 . star formation follows the gas with no substantial trigger from a density wave; 3 . the gas and intermediate-age stars are compressed more in a density wave than the old stars, and 4 . the clouds are larger inside density-wave arms than they are between the arms. Points 1 and 4 are based on observations, point 3 follows from the theory of density waves and point 2 may be considered as an assumption for now, although future observations may verify it directly (cf. §5).

The most important piece of new information that undermines the hypothesis of density-wave-triggered star formation is that spiral arms are bright because density waves are intrinsically strong. No star formation trigger is needed to produce bright spiral arms. The enormous strength of density waves is evident from photographs in the I band (Schweizer 1976; Elmegreen 1981) and from surface photometry (Schweizer 1976; Strom, Jensen and Strom 1976; Elmegreen and Elmegreen 1984, 1985; Kennicutt and Edgar 1986).

The observed blueness of spiral arms also requires no star formation trigger. Azimuthal variations in the blue intensity of a galaxy are only slightly larger than the azimuthal variations in the R or I bands (Strom, Jensen and Strom 1976; Schweizer 1976; Jensen 1977; Elmegreen and Elmegreen 1984; Kennicutt and Edgar 1986). The excess blueness is so slight that it can usually be explained as the combination of a greater compression of gas compared to stars, with star formation merely following the gas, and a greater compression of intermediate-age stars than old stars (which is expected because of the difference in the velocity dispersion and scale height for the different stellar ages).

The age and color gradients expected for density wave arms are rarely found in galaxies (Schweizer 1976; Talbot, Jensen and Dufour 1979; Humphreys and Sandage 1980). This was explained to be the result of epicyclic mixing of clouds (Bash 1979) or stars (Yuan and Grosb $\varnothing 1$ 1981) behind the density wave front, or the result of propagating star formation (Gerola and Seiden 1979; Elmegreen 1979; Smith et al. 1984) behind the front. The lack of such gradients can also be explained by star formation that follows the gas, with no density wave trigger. Then the few age gradients that do appear could be the result of erroneous age estimates (such as the claim that HI clouds are younger than HII regions, or that small OB associations are younger than large OB associations), random juxtapositions of differentaged regions, or propagating star formation. The age gradient found in M31 by Efremov (1985), for example, could result from an incorrect determination of the position of the "edge" of the arm, which is assumed to be the time-zero point for star formation. The appearance of an edge which separates the "younger" gas from the "older" stars could be entirely the result of extinction in the dust and molecular clouds that are known to lie on the gas side. The proposed age gradient would then be an illusion created by a local displacement between the gaseous density peak and the stellar density peak in the wave (such displacements are expected, as discussed below with regard to shocks). Stellar distributions in infrared images of density waves rarely have edges as sharp as the one discussed by Efremov.

The observed streaming motions of stars at their extrapolated times of birth have been interpreted as evidence for the triggering hypothesis (Grosb $\varnothing 1$ 1977). In fact, the flow pat- 


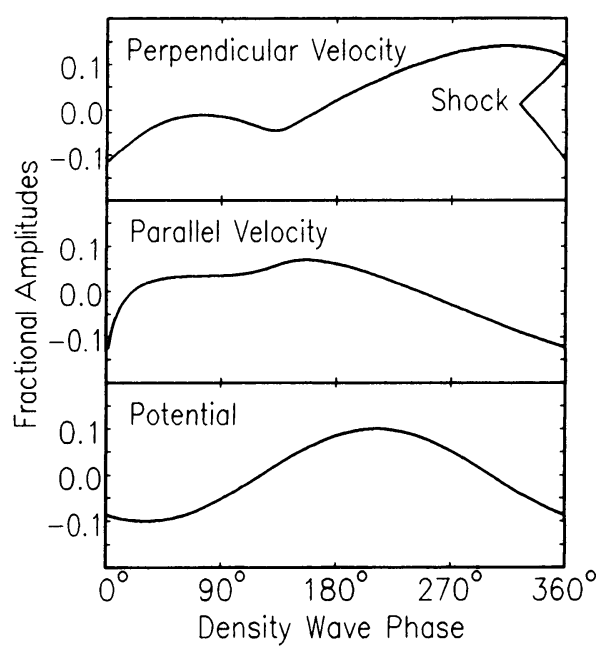

(a)

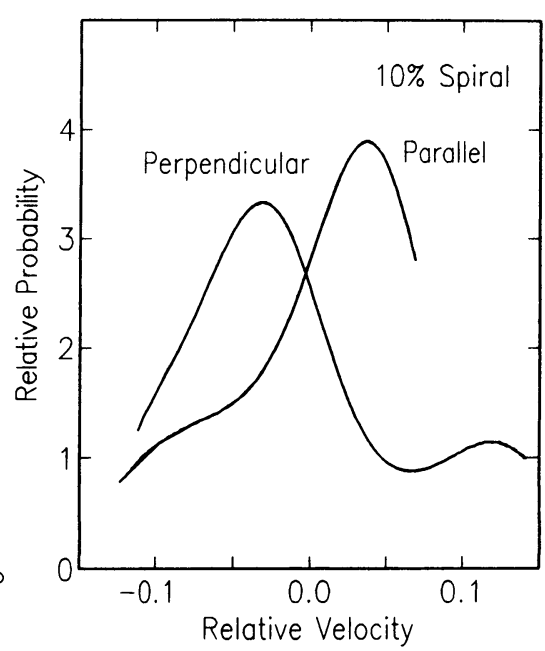

(b)

Figure 3: (a) The perturbed flow patterns (parallel and perpendicular components) for a uniform gas in a density wave with the indicated perturbed potential is shown as a function of the spiral coordinate normal to the wave. The units of velocity are the equilibrium circular velocity. (b) The probability distribution functions for perpendicular and parallel components are shown. The shift in the peak probability from zero velocity indicates a preference for streaming motions of newborn stars, even without density-wave triggered star formation.

tern of gas in a density wave would produce such streaming motions even if star formation were random in time. Figure $3 a$ illustrates the perturbed flow pattern for gas in a density wave, calculated from equations in Roberts (1969) for a 10\% spiral arm potential (EE86). The unit of the perturbed velocity is the equilibrium circular rotation speed, $v_{o}$. Figure $3 \mathrm{~b}$ shows the probability distribution function for the velocity components parallel and perpendicular to the spiral arms, calculated in the case of the parallel flow from the equation

$$
P_{\|}(v)=\int_{-\infty}^{\infty} P\left(v \mid v_{\|}\right) P\left(v_{\|}\right) d v_{\|},
$$

where the conditional expectation of the velocity $v$, given a mean flow velocity $v_{\|}$, is taken to have a Gaussian distribution,

$$
P\left(v \mid v_{\|}\right) \propto \exp \left\{-\left(v-v_{\|}\right)^{2} / 2 \sigma^{2}\right\},
$$

with $\sigma=10 \mathrm{~km} \mathrm{~s}^{-1}$. The flow probability distribution, $P\left(v_{\|}\right) d v_{\|}$, is converted to time coordinates, and then spiral-wave angle coordinates, as follows:

$$
P\left(v_{\|}\right) d v_{\|}=P(t) d t=P_{o} d t=P_{o} \frac{d \theta}{d \theta / d t}=P_{o} \frac{d \theta}{\left(v_{\perp}+v_{o} \perp\right) / R} .
$$

The time-dependent probability function has been sei equal to a constant, $P_{o}$, as if the star formation rate is constant in time for each parcel of gas (and not triggered by the density wave). The velocity component perpendicular to the spiral is denoted by $v_{\perp}$. Figure $3 \mathrm{~b}$ in- 
dicates that the peaks of the probability distribution functions are shifted toward streaming motions parallel to the arms and into the galactic center. No net flux of gas occurs because $\int v P(v) d v=0$ for both components.

The Schmidt law of star formation is also subject to modern revisions. The original density-squared law was determined from observations of only the atomic gas density (e.g., Hamajima and Tosa 1975), whereas star formation is now thought to depend primarily on the molecular gas density. In fact, the star formation rate per molecule appears to be approximately constant, both with radius in a galaxy (Talbot 1980; Young and Scoville 1982a; Scoville and Young 1983; Solomon 1983; DeGioia-Eastwood et al. 1984), and from galaxy to galaxy within a specific Hubble type (Young and Scoville 1982b). Thus the modern version of the Schmidt law suggests that, on a large scale, star formation follows the molecular gas, with no substantial increase in the star formation rate per unit molecular mass at higher mean molecular gas densities. (The star formation rate still depends sensitively on the local density over the small scales of individual clouds, e.g., Viallefond, Goss and Allen 1982).

The observation that the arm/interarm contrast for molecules exceeds that for atoms appears to support the triggering hypothesis. There are substantial uncertainties in the arm/interarm ratio for the atomic mass density, however, because of the possibility that the $\mathrm{HI}$ in spiral arms is optically thick (see, for example, Dickey and Benson [1982] or Kulkarni [1983] for observations of large HI opacities in the inner Galaxy). Moreover, the HI arm/interarm contrast could be slightly less than the $\mathrm{H}_{2}$ contrast simply because the density-wave compression of the $\mathrm{HI}$ fluid, which has a high velocity dispersion and high scale height, could be slightly less than the compression of the $\mathrm{H}_{2}$ fluid, which has a low velocity dispersion and low scale height. The greater temperatures of molecular clouds in spiral arms (Solomon, Sanders and Rivolo 1984) also need not indicate a preferential triggering of star formation there, because the observed temperature variation could be the result of a change in mean cloud mass ( $\$ 2.4)$, accompanied by the usual variation of the maximum star mass with cloud mass (Larson 1982; Güsten and Mezger 1983; Elmegreen 1983).

The connection between spiral arm shocks and star formation is vague in most galaxies. The dust lanes are usually irregular, and they are sometimes separated from the following star formation sites by clear, inactive regions. Spiral arm shocks do exist in galaxies, but there is no compelling reason to believe that such shocks generally trigger star formation, although such triggering may be possible in exceptional cases ( $\$ 2.5)$. DeGioia-Eastwood et al. (1984) found little correlation between the shock strength and star formation rate in NGC 6946. Moreover, the simultaneous occurrence of both smooth interstellar shock fronts (dust lanes) on the inner edges of density-wave potential minima, and conglomerates of starforming clouds right at the potential minima, downstream from the shock, suggests that different types of interstellar gas can react to density waves in different ways. The diffuse component (i.e., the warm intercloud medium and/or the diffuse clouds) may shock coherently, and some of the shocked matter may form low-density molecules (Allen $e$ al. 1985; Ichikawa et al. 1987), whereas the dense, cloudy component (i.e., the strongly selfgravitating molecular clouds) may follow the stars, moving in a ballistic fashion (e.g., Bash et al. 1977). Star formation may follow only the dense component and be independent of the shock in the diffuse component. In fact, the pressure inside a giant molecular cloud usually exceeds the typical galactic shock pressure by a factor of 10 or more, so the starforming core of such a cloud should not be significantly affected by a passing shock front.

Further evidence for the density-wave triggering of star formation formerly came from the explanation of the Luminosity Class offered by Roberts, Roberts and Shu (1975). These authors suggested that Luminosity Class I galaxies have thinner-looking spiral arms and greater luminosities than Luminosity Class II through V galaxies because the shock strength is strongest, and the star formation rate presumably largest, in Luminosity Class I galaxies. Such a variation of shock strength is indeed likely, based on the observed variation of the 
rotation speed, but the luminosity of a galaxy depends almost entirely on the galaxy size (EE86). The surface brightnesses of galaxies are nearly constant (Freeman 1970) so the average star formation rates per unit area must be nearly constant also.

The Luminosity Class also depends somewhat on whether or not the galaxy contains a density wave. The existence of a wave can depend on gas content (and therefore, star formation rate) for reasons that are independent of direct star formation triggers (reasons such as gaseous damping of wave energy [Kalnajs 1972; Roberts and Shu 1972 ], gaseous contributions to the self-gravity of the wave, or gaseous contributions to the density of stars with low velocity dispersions via wave-independent star formation [Sellwood and Carlberg 1984]). Thus spiral arm quality, as measured by the Luminosity Class (and determined partially by the strength of a density wave but not necessarily by the strength of a shock) could, in principle, correlate with galaxy surface brightness (as determined by the star formation rate) because both the spiral wave amplitude and the star formation rate depend on the gas density (but not directly on each other).

The origin of the Luminosity Class may be related to the observation that the arm thickness in a galaxy depends more on the flow-through velocity in the arm than on the shock strength (Kennicutt and Hodge 1982). This observation, combined with the fact that the Luminosity Classification System is primarily a sequence of galaxy size, suggests that spiral arms look relatively thin in Luminosity Class I galaxies because the rest of the galaxy is relative large.

The final piece of evidence for the triggering hypothesis, as discussed above, is the presence of radial metallicity gradients and star-formation-rate gradients. These observations have other interpretations, however. For example, radial metallicity gradients have been modelled without density waves by Diaz and Tosi (1984), Lacey and Fall (1985) and Chiosi and Matteuchi (1985). Besides, similar metallicity gradients occur in galaxies with and without density waves. NGC 7793 (Edmunds and Pagel 1984; McCall 1982) and several other galaxies studied by McCall (1982; e.g., NGC 4736, NGC 5055, NGC 2403, NGC 4395 and NGC 4449) have no evidence for a density wave, but they contain metallicity gradients similar to those in grand design galaxies of the same Hubble types. Metallicity appears to depend primarily on galaxy mass or surface density (Pagel and Edmunds 1981; McCall 1982; Bothun et al. 1984).

Radial gradients in the star formation rate have also been modelled without a density wave. The theory of stochastic self-propagating star formation discussed by Seiden (1983) produces a radial variation in the star formation rate in a galaxy with a flat rotation curve that is approximately proportional to $1 / R-C$ for radius $R$ and constant $C$ (Seiden et al. 1984). This is the same expression as the density wave interaction frequency, $\Omega-\Omega_{P}$, for a flat rotation curve. Both of the constants $C$ and $\Omega_{P}$ are chosen so that the star formation rate approaches zero at the outer edge of the disk. The theory proposed by Dopita (1985) also obtains the observed formation rate gradients without density waves.

\subsection{Do Density Waves Trigger Supercloud Formation?}

The HII regions and molecular clouds in density-wave spiral arms tend to be larger than the HII regions and molecular clouds between the arms (Mezger 1970; Georgelin and Georgelin 1976; Stark 1979; Kennicutt and Hodge 1980; Rumstay and Kaufman 1983; Cohen et al. 1985; Dame et al. 1986). Thus, density waves do something for star formation, even if they do not greatly enhance the overall star formation rate. They may, for example, stimulate the coagulation of small clouds into large clouds.

The large clouds that are observed in spiral arms often have masses in excess of $10^{7}$ $\mathbf{M}_{\odot}$. These clouds are much more massive than Orion-type "giant molecular clouds," which sometimes appear only as cores inside the larger structures. We refer to the largest regions 
as superclouds. McGee and Milton (1964) studied giant HI clouds along four spiral arm segments in the outer part of the Galaxy. Each segment was found to contain between 5 and $12 \mathrm{HI}$ clouds with masses of approximately $10^{7} \mathrm{M}_{\odot}$ (also apparent in more recent maps by Henderson, Jackson anci Kerr 1982 and Grabelsky 1985). The local distribution of giant HI clouds, corresponding to the local spiral arm "shingles", was discussed by Quiroga and Schlosser (1977) and Schlosser and Görnandt (1984). Clouds with $10^{7} \mathbf{M}_{\odot}$ of molecules have also been identified in the Sagittarius -Carina spiral arm (Cohen et al. 1985; Dame et al. 1986), and two kiloparsec-size $\mathrm{OH}$ complexes in the Perseus arm were mapped by Wouterloot (1981). Equally large clouds appear as dust complexes in optical photographs of galaxies (Elmegreen 1980).

Giant HI clouds are clearly visible in aperture synthesis maps of nearby spiral galaxies. Table 1 gives the average minimum masses of the largest HI clouds in six galaxies, the average minimum HI column densities, and the critical column densities for self-gravitational binding against galactic tidal forces. The HI masses and column densities were determined from published HI temperature contours; they are lower limits to the total cloud masses and column densities if the $\mathrm{HI}$ is optically thick or the gas is partially molecular. The tidal limits for the column densities were determined from the tidal limits for the space densities, which equal approximately $A \Omega / G$ for Oort rotation constant $A$ and angular velocity $\Omega$, multiplied by an estimated cloud thickness of $200 \mathrm{pc}$. The observed column densities exceed the tidal limits, suggesting that the clouds are gravitationally bound, provided their internal velocity dispersions are not too large (Stark and Blitz 1978; Blitz and Glassgold 1982). The typical mass is several times $10^{7} \mathrm{M}_{\odot}$.

Table 1: HI SUPERCLOUDS IN NEARBY GALAXIES

\begin{tabular}{cccccc}
\hline Galaxy & $\begin{array}{c}\text { Average } \\
\text { Cloud Mass } \\
\left(\times 10^{7} \mathrm{M}_{\odot}\right)\end{array}$ & $\begin{array}{c}\text { Number of } \\
\text { Clouds } \\
\text { Measured }\end{array}$ & $\begin{array}{c}\mathrm{N}(\mathrm{HI}) \\
\left(\times 10^{21} \mathrm{~cm}^{-2}\right)\end{array}$ & $\begin{array}{c}\mathrm{N}_{\text {crit }} \\
\left(\times 10^{21} \mathrm{~cm}^{-2}\right)\end{array}$ & Ref. \\
\hline M33 & 0.3 & 5 & 2 & 1 & 1 \\
M31 & 3 & 4 & 3 & 1 & 2,3 \\
M106 & 3 & 7 & 2 & 0.3 & 4 \\
M101 & 7 & 5 & 2 & 0.4 & 5,6 \\
M81 & 0.6 & 6 & 2 & 2 & 7 \\
IC 342 & 3 & 10 & 1 & 0.2 & 8 \\
\hline
\end{tabular}

References: 1. Newton 1980a; 2. Unwin 1980a,b; 3. Bajaja and Shane 1982;

4. van Albada 1980; 5. Allen and Goss 1979; 6. Bosma, Goss and Allen 1981;

7. Rots $1975 ; 8$. Newton 1980 b.

What is the formation mechanism for such giant gas complexes? Because the interstellar medium is generally 'nhomogeneous, superclouds probably form by the agglomeration of smaller clouds. The clouds that enter a spiral arm may coalesce into giant complexes (Casoli and Combes 1982; Roberts and Hausman 1984; Hausman and Roberts 1984; Combes and Gerin 1985; Tomisaka 1984, 1985; Fukunaga and Tosa 1987). The complex of clouds studied by Pérault, Falgarone and Puget (1985) may be an example of such an agglomeration, although on a smaller scale. Because the mass of a supercloud is comparable to the expec.ed Jeans mass in a galactic disk, gravitational instabilities may also be important (Elmegreen 1979; Cowie 1981; Elmegreen and Elmegreen 1983b; Jog and Solomon 1984; Tomisaka 1985; Balbus and Cowie 1985). For an axisymmetric instability in a sheared medium, the wavenumber of the fastest growing mode is $k=\pi G \sigma / c^{2}$ for one-dimensional rms 
velocity dispersion $c$, and mass column density through the disk $\sigma$. The Jeans mass is thus $\sigma \pi(\pi / k)^{2}=\pi c^{4} / G^{2} \sigma$, which is on the order of $10^{7} \mathrm{M}_{\odot}$ for $c \simeq 5 \mathrm{~km} \mathrm{~s}^{-1}$ and $\sigma \simeq 10 \mathrm{M}_{\odot} \mathrm{pc}^{-2}$ (corresponding to a midplane density of $1.5 \mathrm{~cm}^{-3}$ and a full disk thickness of $200 \mathrm{pc}$ ). Thus, the collisional agglomeration of small clouds could be influenced, or driven, by selfgravitational forces, possibly leading to a characteristic cloud mass equal to the Jeans mass. Magnetic forces in the Parker instability are probably less important than self-gravity (Elmegreen 1982a).

Superclouds probably form in galactic disks that are generally stable against catastrophic self-gravitational collapse of axisymmetric perturbations. Such a disk has $\pi G \sigma / \kappa c$ $<1$ for epicyclic frequency $\kappa$ (Safronov 1960; Toomre 1964). Without such stability, star formation would be too rapid. The formation of superclouds could then follow an episode of gas compression, as in a density wave, which temporarily increases $\sigma$ enough to trigger the axisymmetric instability (Balbus and Cowie 1985). Alternatively, it could continuously operate in a stable disk, possibly by the mechanism of shear amplification discussed by Goldreich and Lynden-Bell (1965) or Hunter and Horak (1983). Non-axisymmetric instabilities could also grow in a gas disk that is axisymmetrically stable (Morozov 1985).

\subsection{Galaxies Where Density Waves Do Trigger an Excess of Star Formation}

Density waves may directly trigger star formation in some regions of galaxies, such as the nuclei of barred galaxies that have strong infrared emission (Mountain et al. 1987), or near the ends of the bars of barred galaxies, where star formation is often very prominent. In the first case, cloud formation may be triggered by the strong spiral shock that forms between two inner Lindblad resonances (Sanders and Tubbs 1980). Barred galaxies with nuclear spirals, such as NGC 4314 (Sandage 1961; Wakamatsu and Nishida 1980), appear to have shocks of this type. In the second case, cloud formation may be triggered by the strong shock that occurs near the end of the stellar bar (Sanders and Huntley 1976; Liebovitch and Lin 1978). Other regions of density-wave triggered star formation are possible too, including a small amount of direct triggering in normal galaxies.

The difference between regions where density waves trigger star formation and regions where they do not is a mystery. One possible difference is in the degree of development of the large-scale gravitational instability thought to be involved with supercloud formation. Weak shocks may provoke only cloud coagulation without any instabilities, or only slowlygrowing instabilities, or low-amplitude "swing amplification" rather than true instabilities, with the result that pre-existing cloud centers merely move closer together, forming complexes without significantly changing the star formation rate per unit gas mass. Strong shocks could provoke more rapid instabilities, or true instabilities in an otherwise stable environment. Then the self-gravitational collapse of the gas might be catastrophic, and the star formation rate per unit gas mass could increase. The distinction between weak and strong shocks in this sense depends critically on the rate of dissipation of energy inside a growing perturbation. If the gravitational binding energy of a supercloud does not dissipate fast enough, then the average density inside the cloud will remain low throughout its life, which is probably limited in this case by the galactic tidal forces that disrupt it when it emerges from the density-wave crest. Such slowly dissipating clouds might not be expected to enhance the star formation rate very much. If the dissipation is rapid, however, then the mean cloud density can increase enormously, strongly-bound molecular cores can form, and galactic tidal forces may not disrupt them. The triggering of an excess of star formation in density-wave shocked gas presumably depends on the formation of such new cores.

The cloud-cloud collisions that accompany supercloud formation may not directly trigger an excess of star formation (Stone 1970; Lattanzio et al. 1985), although these col- 
lisions could eventually lead to gravitational collapse if the total mass of the combined cloud exceeds the Lane-Emden critical mass for the external pressure.

\section{GIANT REGIONS OF STAR FORMATION IN GALAXIES}

Star formation in normal spiral galaxies often operates coherently on scales of several hundred parsecs to a kiloparsec, and is sometimes associated with a total gas mass of $10^{7} \mathrm{M}_{\odot}$ or more. Even the separations between star formation sites in a galaxy give evidence for the operation of a large-scale coherent process during star formation (Elmegreen and Elmegreen 1983b; Braunsfurth and Feitzinger 1985). Ionization from the largest regions can dominate the total thermal radio emission from a galaxy (Mezger 1970; Israel 1980; Hunter 1982), and the associated molecular clouds can dominate the total $\mathrm{CO}$ and infrared emission (Solomon and Sanders 1980; Caux et al. 1984, 1985; Myers et al. 1986; Dame et al. 1986). The largest HI clouds in a galaxy are of ten associated with these star forming regions (McGee and Milton 1966; Wright 1971; Allen et al. 1973; Emerson 1974; Unwin 1980b; Page and Carruthers 1981; Viallefond et al. 1982, 1983; Venger et al. 1984), although the atomic gas is sometimes located to the side of the star-forming core (Boulesteix et al. 1974; Newton 1980a,b; Unwin 1980a; Viallefond et al. 1981). Molecules have been observed in or near some of the giant HI clouds in the Carina arm of the Galaxy (Grabelski 1985), and in M101 (Blitz et al. 1981) and M31 (Boulanger et al. 1981, 1984; Linke 1982; Stark 1985; Ichikawa et al. 1985).

Giant HII regions are of ten excited in several cores where star formation seems to occur simultaneously (Israel et al. 1975; Kennicutt and Hodge 1980; Comte and Duquennoy 1982; Viallefond et al. 1983). The emission line widths are sometimes broad, possibly as a result of virialized motions in a self-gravitating complex (Terlevich and Melnick 1981), and possibly as a result of strong stellar winds and champagne flows (Dyson 1979; Skillman and Balick 1984; Gallagher and Hunter 1983). Of course, both pressure-driven and virialized motions could occur simultaneously. The winds and HII regions could generate the turbulence that supports a large complex, so these pressures could determine its radius inside the self-gravitational potential well. Then the turbulence will be virialized automatically.

The Galactic HII regions NGC 3372 (Carina), NGC 3603 and W49 are typical of the largest regions in galaxies (Kennicutt 1984; Persi et al. 1985; Moffat et al. 1985), although other galaxies can have HII regions much larger than any in our Galaxy. The giant HII region and cluster NGC 604 in the galaxy M33, which is excited by the equivalent of 60 O5 stars, contains several wind-blown shells superposed on a systematic outflow of ionized gas from a 350 pc-size "blister" on a nearby, 2 kpc-size HI cloud (Rosa and Solf 1984; Hippelein and Fried 1984; Sanz Fernández de Córdoba and Benvenuti 1984). The HII region and cluster NGC 5471 in M101 is like NGC 604, although NGC 5471 contains a supernova remnant in addition (Skillman 1985). Molecular clouds are present near both NGC 604 (Blitz 1985) and NGC 5461 in M101 (Blitz et al. 1981). Similar regions, sometimes called "hot spots" in galaxies, have been discussed recently by Terlevich (1984), Skillman and Balick (1984), Rosa et al. (1984), Kennicutt and Hodge (1984), Melnick et al. (1985), Durret et al. (1985), Wynn-Williams and Becklin (1985), and Arp and Sandage (1985). The most active regions of star formation in irregular galaxies have a similar scale (Gallagher and Hunter 1984).

The stellar groupings that result from large-scale star formation have been observed in many forms. The most luminous concentrations of massive stars have been termed superassociations (Ambartsumian et al. 1963; Shahbazian 1968, 1970); recent studies are by Dolidze (1981), Condon (1983), Heidmann (1983), Petrosyan et al. (1984a,b,c) and Khachikian (1984). Somewhat smaller regions are star complexes, which are concentrations of Cepheid variables and red supergiants typically 150 to 1000 parsecs in size. Star com- 
plexes often contain one or more $\mathrm{OB}$ associations and clusters with stellar ages between 10 and 100 million years (Efremov 1982b). They have been identified in the Milky Way (Efremov 1978, 1979), M33 (Efremov 1982b), M31 (Efremov 1980b, 1982a) and the Large Magellanic Cloud (Efremov and Pavlovskaya 1982). The local example of large-scale star formation is Gould 's Belt, which has a size comparable to a superassociation ( $1 \mathrm{kpc})$. Gould's Belt appears primarily as a local concentration of B stars (Lesh 1968; Stothers and Frogel 1974), but similar stellar concentrations, sometimes consisting of A stars, are also found elsewhere ( Kevanishvili 1969, 1975; Moore and FitzGerald 1973). More distant examples of Gould's Belt are the spiral arm shingles observed by Schmidt-Kaler and Schlosser (1973), and discussed more recently by Brosche and Schwan (1984). Giant star-forming regions in our galaxy have also been cataloged by Shevcheko $(1979,1980)$. For example, the HII regions, M16, M17, M8 and M20 appear to be part of a single star-forming region (Stal'bovskii and Shevchenko 1981). Stellar streams or superclusters (Eggen 1965; Latyshev 1980), and coeval galactic clusters (Barkhatova and Pavlovskaya 1981; Lyngå and Wramdemark 1984) may be examples of the final remnants of large-scale star formation.

The formation of stars on a scale of several hundred parsecs to a kiloparsec could imply either that the primordial clouds are a kiloparsec in size (cf. $\S 2.4$ ), or that star formation continuously propagates from one generation to another $(\S 4)$. Simulations of propagating star formation (Seiden and Gerola 1982) suggest that kiloparsec-size patches of activity should be common in galaxies, even without coherent cloud complexes to define the basic scale, and even when the propagation step size is only 50 to 100 parsecs. This patch size is the correlation length for the percolation phase transition (Seiden, private communication).

\section{LARGE-SCALE PROPAGATING STAR FORMATION}

The concept of propagating star formation originated with Öpik (1953) and Oort (1954), who proposed that the expansion of OB associations (Ambartsumian 1947) results from star formation in fast-moving shock fronts. Pressure-induced collapse of individual globules was also considered as a possible trigger for star formation by Bok (1955) and Dibai (1958). Propagation on a galactic scale was discussed qualitatively by Baade (1963) for IC 1613, and in some detail by Westerlund and Mathewson (1966) for the Large Magellanic Cloud. The first extensive evidence for propagating star formation was presented by Blaauw (1964), who showed that $\mathrm{OB}$ associations sometimes exhibit a linear sequence of stellar ages. More recent evidence for propagation followed the discovery of molecular clouds. A compilation made in December 1984 listed 27 small-scale regions and 8 large-scale regions for which some kind of propagation had been proposed (Elmegreen 1985a). Table 2 summarizes the properties of 10 regions of proposed large-scale propagation.

Large-scale propagating star formation has a spectacular example in the solar neighborhood. The first generation of stars appears to have been the Cas-Tau group (Blaauw 1956,1984). The pressure from this group organized the local gas into a giant expanding ring (Lindblad 1967; Hughes and Routledge 1972; Lindblad et al. 1973). The second generation is now along the periphery of the ring, including the Ori OB1, Per OB2, Sco-Cen and Lac OB1 associations (Olano 1982; Elmegreen 1982b).

Mechanisms for propagation over distances of several hundred parsecs or more are not well understood because the observations of such propagation are scarce. One possibility is that the pressure from a first generation $O B$ association pushes the ambient interstellar gas into an expanding shell, which eventually becomes gravitationally unstable and fragments into second generation clouds (Olano 1982; Elmegreen 1985b). Also possible is the compression of pre-existing clouds in the region surrounding the first generation $\mathrm{OB}$ association (Dopita et al. 1985). The likely driving force for these mechanisms is the energy from 
Table 2: REGIONS OF PROPOSED LARGE-SCALE PROPAGATION

\begin{tabular}{|c|c|c|c|c|c|}
\hline \multirow[t]{2}{*}{ Region } & \multirow{2}{*}{$\begin{array}{l}\text { Diameter } \\
\text { (parsecs) }\end{array}$} & \multirow{2}{*}{$\begin{array}{l}\text { Propagation } \\
\text { Vel. }\left(\mathrm{km} \mathrm{s}^{-1}\right)\end{array}$} & \multicolumn{2}{|c|}{ 2nd Generation } & \multirow[t]{2}{*}{ Ref } \\
\hline & & & Nur & nber Names & \\
\hline Const.III (LMC) & 2000 & 36 & 18 & $\begin{array}{l}\text { LH51-55,60,63,75, } \\
\text { LH76,78,79,82,83, } \\
\text { LH86,88,91,92,95 }\end{array}$ & 1,2 \\
\hline LH77 (LMC) & 2000 & 30 & 7 & $\begin{array}{c}\text { N51 N57 N59 N64 } \\
\text { N63 N55 N50 }\end{array}$ & 3 \\
\hline LH85, 89 (LMC) & 200 & 20 & 1 & LH90 & 4 \\
\hline N158 (LMC) & 180 & 20 & 1 & & 4 \\
\hline Shells in LMC & $100-1000$ & $<30$ & $5-20$ & & 5 \\
\hline SMC X-1 (SMC) & 200 & 35 & 2 & N84A N83A & 4 \\
\hline Origem Loop & 120 & 20 & 5 & $\begin{array}{c}\text { S254 S258 S261 } \\
\text { S259? S269? }\end{array}$ & 6,7 \\
\hline Cyg X & 100 & 15 & 2 & IC1318a HS191 & 8 \\
\hline Lindblad Ring & 600 & 10 & 4 & $\begin{array}{l}\text { Ori OB1 Per OB2 } \\
\text { Sco-Cen Lac OB1 }\end{array}$ & 9,10 \\
\hline Mon OB2 & 250 & 20 & 1 & Rosette Neb & 11 \\
\hline
\end{tabular}

References: 1. Westerlund and Mathewson 1966; 2. Dopita et al. 1985; 3. de Boer and Nash 1982; 4. Lortet et al. 1987; 5. Meaburn 1980; 6. Berkhuijsen 1974; 7. Pismis and Hasse 1982; 8. Dixon et al. 1981; 9. Olano 1982; 10. Elmegreen 1982b; 11. Gosachinskij and Khersonskij 1982.

massive stars. Regions of massive star formation receive so much energy from starlight, stellar winds and supernova explosions that the local gas pressure can be 10 to 100 times larger than it is in the ambient medium for a period of $10^{7}$ years. This pressure may trigger the collapse of previously stable clouds in the vicinity, and at the same time drive an expansion of these clouds and other gas away from the association at a velocity of $\sim 10$ to 50 $\mathrm{km} \mathrm{s}^{-1}$, or more. By the time the high pressure subsides, the disturbance may have grown to a radius of 100 parsecs or more. Thereafter the swept-up material will conserve momentum. When the expansion finally slows to the local sound speed and the swept-up gas begins to disperse, the diameter of the disturbance may exceed 500 parsecs. Disturbed regions are so large compared to the mean separation between $\mathrm{OB}$ associations that they may overlap, in which case $\mathrm{OB}$ associations would directly influence the energetics of nearly all of the interstellar matter in a galaxy.

Evidence for such large-scale, pressure-driven expansion is all around us, as giant loops and shells around the local OB associations (Weaver 1979; Cowie et al. 1979, 1981), and as the ring of expanding gas (Lindblad 1967) centered on the old Cas-Tau association (Olano 1982). Many other giant HI shells in our Galaxy (Brand and Zealey 1975; Heiles 1979; Bochkarëv 1985) and in other galaxies (Hayward 1964; Westerlund and Mathewson 1966; Hodge 1967; Hindman 1967; Allen, Goss and van Woerden 1973; Brinks and Bajaja 1983), and giant ring-like HII regions in other galaxies (Gum and de Vaucouleurs 1953; Meaburn 1980; Boulesteix, et al. 1974; Pellet, et al. 1978; Courtès, et al. 1981; Dettmar et al. 1984) could also have formed by pressures from young stars.

The far-reaching influence of massive star formation on interstellar matter has led to theoretical considerations of sequential star formation (Ögelman and Maran 1976; Elmegreen and Lada 1977; Herbst and Assousa 1977), self-propagating star formation in galaxies 
(Mueller and Arnett 1976; Gerola and Seiden 1978) and self-regulated star formation (Cox 1983; Franco and Shore 1984). It has also revealed non-linear phenomena in global starformation processes, analogous to percolation phase transitions (Seiden et al. 1979; Seiden and Gerola 1982), limit-cycle oscillations (Seiden et al. 1982; Nepveu and Robnik 1984), and feedback control (Talbot and Arnett 1975; Seiden et al. 1979, 1984; Shore 1981, 1983; Tomisaka et al. 1981; Ikeuchi et al. 1984; Seiden 1983; Struck-Marcell and Scalo 1984; Bodifée and de Loore 1985; Chiang and Prendergast 1985; Dopita 1985).

Propagating star formation leads to mutual interactions between cloud formation, star formation and cloud destruction. Non-linear models that simulate such interacting systems can be deceptively simple for some parameters, but they can exhibit strong oscillations or explosions when slightly different parameters are used. The more exotic modes of star formation have been considered for galactic nuclei (Loose et al. 1982; Loose and Fricke 1982a,b), for spatially confined systems, such as dwarf galaxies (Gerola et al. 1980; Comins 1984) or galaxies where the post-star-formation relaxation time of the gas exceeds the propagation time through the disk (Seiden et al. 1982), and for proto-galaxies (Larson 1980; Cox 1983, 1985). Starburst galaxies (Weedman et al. 1981; Gehrz et al. 1983; Balzano 1983; Telesco 1984), clumpy irregular galaxies (Casini and Heidmann 1976; Benvenuti et al. 1982; Boesgaard et al. 1982) and extragalactic HII regions (Sargent and Searle 1970) could also be going through temporary phases of high activity in which propagating star formation plays an essential role.

Propagating star formation can significantly affect the appearance of a normal galaxy. Theoretical models of such propagation show relatively long spiral arms (Gerola and Seiden 1978; Seiden and Gerola 1979; Cowie and Rybicki 1982; Balbus 1984; Freedman and Madore 1984; Nozakura and Ikeuchi 1984), with realistic pitch angles (Schlosser and Musculus 1984) and spiral arm spurs (Feitzinger and Schwerdtfeger 1982). The highpressure cavities created in the models resemble the HI supershells observed in galaxies (Feitzinger and Seiden 1983). Brightness profiles of the model disks also match those of real galaxies (Seiden et al. 1984). Such models do not, however, produce red and symmetric spiral arms, which must be density waves. Propagating star formation presumably operates in gaseous disks that also contain density waves (Gerola and Seiden 1979; Kaufman 1981; Smith et al. 1984).

\section{FUTURE OBSERVATIONS}

The extent to which star formation is triggered by normal density waves must be determined by observations, but this may be very difficult to do. One desired quantity is the local star formation rate per unit gas mass $\left(\mathrm{H}+\mathrm{H}_{2}\right)$, which should be plotted as a function of density wave phase. Azimuthal variations in the star formation rate can be determined, in principle, from observed variations in the flux of thermal radio continuum, $\mathbf{H} \alpha$, ultraviolet, or farinfrared radiation, unless the initial stellar mass function varies with density wave phase (as suggested by Güsten and Mezger 1983). If the initial mass function varies, then the fluxes will not uniquely determine the star formation rates. The total gas mass might be determined from the $\mathrm{CO}$ and $\mathrm{HI}$ fluxes. The $\mathrm{CO}$ to $\mathrm{H}_{2}$ conversion is uncertain however, and could vary with density wave phase if the mean cloud properties vary. The HI mass is also uncertain, because of unknown opacities. Variations in the total gas mass might be better determined from variations in the far infrared dust emission, unless the dust-to-gas ratio also varies with phase.

If azimuthal variations in the star formation rate per unit gas mass can be determined accurately, then the phase-dependent component of the amplitude of these variations should 
indicate the extent of density-wave triggering. Correlations between this amplitude and the galaxy Hubble type, stellar arm amplitude, shock strength, and so on, would be interesting.

Global star formation rates per unit gas mass, or per unit area, should be compared for galaxies with and without density waves (cf. $\S 2.2$ ). Total star formation rates, not normalized to galaxy area or gas mass, are not as useful as the normalized quantities because of the large variation in galaxy size for both flocculent and grand design galaxies. The masses and mass distributions of clouds and young clusters should also be compared for these different galaxy types.

If density waves do not commonly trigger star formation, then the corotation radius for the pattern need not occur at the outer radius of the optical disk, as previously believed (Shu, Stachnik and Yost 1971; Roberts, Roberts and Shu 1975). Any determination of the corotation radius in a non-barred galaxy would therefore be very interesting. This radius might be determined from the position where the relative velocity of the spiral arm streaming motion changes sign (Kalnajs 1978).

Long-range propagation can probably be recognized in a large number of regions once observers know what to look for. Table 3 might serve as a guideline. For example, the largest HI shells that are observed in other galaxies are expected to contain HII regions or other evidence for recent star formation located along their periphery. Adjacent clusters of stars might appear with an implied propagation velocity equal to approximately $20 \mathrm{~km} \mathrm{~s}^{-1}$, corrected for galactic shear and peculiar motions.

\section{REFERENCES}

Allen, R.J., Goss, W.M. and van Woerden, H. 1973, Astron.Astrophys., 29, 447.

Allen, R.J. and Goss, W.M. 1979, Astron.Astrophys.Suppl., 36, 135.

Allen, R.J., Atherton, P.D. and Tilanus, R.P.J. 1985, Nature, in press.

Ambartsumian, V.A. 1947, Stellar Evolution and Astrophysics, Armenian Academy of Sciences.

Ambartsumian, V.A., Iskudarian, S.G., Shahbazian, R.K., and Sahakian, K.A. 1963, Bull. Byurakan Obs., $33,1$.

Anderson, S., Hodge, P. and Kennicutt, R. Jr. 1983, Astrophys.J., 265 , 132.

Arp, H. and Sandage, A. 1985, Astron.J., 90, 1163.

Baade, W. 1963, in The Evolution of Stars and Galaxies, ed. C. Payne-Gaposchkin, (Cambridge: Harvard University Press), Chapter 16.

Baade, W. and Mayall, N.U. 1951, in Problems of Cosmical Aerodynamics, (Dayton Ohio: Control Air Document Office), p. 165.

Bajaja, E. and Shane, W.W. 1982, Astron.Astrophys.Suppl., 49, 245.

Balbus, S.A. 1984, Astrophys.J., 277, 550.

Balbus, S.A. and Cowie, L.L. 1985, Astrophys.J., 297, 61.

Balzano, V.A. 1983, Astrophys.J., 268, 602.

Barkhatova, K.A. and Pavlovskaya, E.D. 1981, Astron.Tsirk., 1155 , 4.

Bash, F.N. 1979, Astrophys.J., 233, 524.

Bash, F.N., Green, E. and Peters, W.L., III 1977, Astrophys.J., 217, 464.

Bash, F.N. and Visser, H.C.D. 1981, Astrophys.J., 247, 488.

Benvenuti, P., Casini, C. and Heidmann, J. 1982, Monthly Not.Roy.Astron.Soc., 198, 825.

Berkhuijsen, E.M. 1974, Astron.Astrophys., 35, 429.

Biermann, P., Kippenhahn, R., Tscharnuter, W. and Yorke, H. 1972, Astron.Astrophys., 19 , 113.

Blaauw, A. 1956, Astrophys.J., 123, 408.

Blaauw, A. 1964, Ann.Rev.Astron.Astrophys., 2, 213.

Blaauw, A. 1984, Irish Astron.J., 16, 141. 
Blitz, L. 1985, Astrophys.J., 296, 481.

Blitz, L., Israel, F.P., Neugebauer, G., Gatley, I., Lee, T.J. and Beattie, D.H. 1981, Astrophys.J., 249, 76.

Blitz, L. and Shu, F.H. 1980, Astrophys.J., 238, 148.

Blitz, L. and Glassgold, A.E. 1982, Astrophys.J., 252, 481.

Bochkarëv, N.G. 1985, Pis 'ma Astron.Zh., 10, 184 (Sov.Astron.Letts. 10, 76).

Bodifée, G. and de Loore, C. 1985, Astron.Astrophys., $142,297$.

Boesgaard, A.M., Edwards, S. and Heidmann, J. 1982, Astrophys.J., 252 , 487.

Bok, B.J. 1955, Astron.J., 60, 146.

Bosma, A., Goss, W.M. and Allen, R.J. 1981, Astron.Astrophys., 93, 106.

Bothun, G.D., Romanishin, W., Strom, S.E. and Strom, K.M. 1984, Astron.J., 89 , 1300.

Boulanger, F., Stark, A.A. and Combes, F. 1981, Astron.Astrophys., 93 , L1.

Boulanger, F., Bystedt, J., Casoli, F. and Combes, F. 1984, Astron.Astrophys., 140 , L5.

Boulesteix, J., Courtès, G., Laval, A., Monnet, G. and Petit, H. 1974, Astron.Astrophys.,37 , 33.

Brand, P.W.J.L. and Zealey, W.J. 1975, Astron.Astrophys., 38, 363.

Braunsfurth, E. and Feitzinger, J.V. 1985, Astron.Astrophys., $144,215$.

Brinks, E. and Bajaja, E. 1983, in Internal Kinematics and Dynamics of Galaxies, IAU Symposium No. 100, ed. E. Athanassoula, (Dordrecht: Reidel), p. 139.

Brosche, P. and Schwan, H. 1984, Mitt.Astron.Ges., 62, 332.

Casini, C. and Heidmann, J. 1976, Astron.Astrophys., 47, 371.

Casoli, F. and Combes, F. 1982, Astron.Astrophys., 110, 287.

Caux, E., Serra, G., Gispert, R., Puget, J.L., Ryter, C. and Coron, N. 1984, Astron. Astrophys., 137, 1.

Caux, E., Puget, J.L., Serra, G., Gispert, R. and Ryter, C. 1985, Astron.Astrophys., $144,37$.

Chance, D., Bally, J. and Elmegreen, B.G. 1986, in preparation.

Chiang, W.H. and Prendergast, K.H. 1985, Astrophys.J., 297, 507.

Chiosi, C. and Matteuchi, F. 1985, preprint.

Cohen, R.S., Cong, H.I., Dame, T.M. and Thaddeus, P. 1980, Astrophys.J.(Letts.), 239, L53.

Cohen, R.S., Grabelsky, D.A., Alvarez, H., Bronfman, L., May, J., and Thaddeus, P. 1985, Astrophys.J.(Letts.), 290, L15.

Combes, F. and Gerin, M. 1985, Astron.Astrophys., 150, 85.

Comins, N.F. 1984, Astrophys.J., 284, 90.

Comte, G. and Duquennoy, A. 1982, Astron.Astrophys., 114, 7.

Condon, J.J. 1983, Astrophys.J.Suppl., 53, 459.

Courtès, G. and Dubout-Crillon, R. 1971, Astron.Astrophys., 11, 468.

Courtès, G., Boulesteix, J. and Sivan, J.-P. 1981, Acad.Sci.Paris, 292, Ser.II, 1521.

Cowie, L.L. 1981, Astrophys.J., 245, 66.

Cowie, L.L., Songaila, A. and York, D.G. 1979, Astrophys.J., 230, 469.

Cowie, L.L., Hu, E.M., Taylor, W. and York, D.G. 1981, Astrophys.J.(Letts.), 250 , L25.

Cowie, L.L. and Rybicki, G.B. 1982, Astrophys.J., $260,504$.

Cox, D.P. 1983, Astrophys.J.(Letts.), 265, L61.

Cox, D.P. 1985, Astrophys.J., 288, 465.

Dame, T.M., Elmegreen, B.G., Cohen, R.S. and Thaddeus, P. 1986, Astrophys.J., in press.

de Boer, K.S. and Nash, A.G. 1982, Astrophys.J., 255, 447.

DeGioia-Eastwood, K., Grasdalen, G.L., Strom, S.E. and Strom, K.M. 1984, Astrophys.J., 278, 564.

Dettmar, R.J., Heidmann, J., Klein, U. and Wielebinski, R. 1984, Astron.Astrophys., 130 , 424.

de Vaucouleurs, G., de Vaucouleurs, A. and Corwin, G.G. Jr., 1976, Second Reference

Catalog of Bright Galaxies, (Austin: The University of Texas Press). 
Diaz, A.J. and Tosi, M. 1984, Monthly Not.Roy.Astron.Soc., 208 , 365.

Dibai, E.A. 1958, Sov.Astron.AJ, 2, 429.

Dickey, J.M. and Benson, J.M. 1982, Astron.J., 87, 278.

Dixon, M.E. 1968, Monthly Not.Roy.Astron.Soc., 140, 287.

Dixon, M.E. 1971, Astrophys.J., 164, 411.

Dixon, K.I., Johnson, P.G. and Songsathaporn, R. 1981, Astrophys.Sp.Sci., 78, 189.

Dolidze, M.V. 1981, Pis'ma Astron.Zh., 7, 666 (Sov.Astron.Letts., 7, 370).

Donas, J. and Deharveng, J.M. 1984, Astron.Astrophys., 140, 325.

Dopita, M.A. 1985, Astrophys.J.(Letts.), 295, L5.

Dopita, M.A., Mathewson, D.S. and Ford, V.L. 1985, Astrophys.J., 297, 599.

Dubout-Crillon, R. 1977, Astron.Astrophys., 56, 293.

Durret, F., Bergeron, J. and Boksenberg, A. 1985, Astron.Astrophys., 143, 347.

Dyson, J.E. 1979, Astron.Astrophys., 73, 132.

Edmunds, M.G. and Pagel, B.E.J. 1984, Monthly Not.Roy.Astron.Soc., 211 , 507.

Efremov, Yu.N. 1970, Astron.Tsirk. No. 579, p.3

Efremov, Yu.N. 1978, Pis'ma Astron.Zh., 4, 125 (Sov.Astron.Letts. 4, 66).

Efremov, Yu.N. 1979, Pis'ma Astron.Zh., 5, 21 (Sov.Astron.Letts. 5, 12).

Efremov, Yu.N. 1980a, Pis'ma Astron.Zh., 6, 275 (Sov.Astron.Letts. 6, 152).

Efremov, Yu.N. 1980b, Pis'ma Astron.Zh., 6, 333 (Sov.Astron.Letts. 6, 184).

Efremov, Yu.N. 1982a, Pis'ma Astron.Zh., 8, 585 (Sov.Astron.Letts. 8, 314).

Efremov, Yu.N. 1982b, Pis'ma Astron.Zh., 8, 663 (Sov.Astron.Letts. 8, 357).

Efremov, Yu.N. 1985, Pis'ma Astron.Zh., 11, 69 (Sov.Astron.Letts. 11, 169).

Efremov, Yu.N. and Ivanov, G.R. 1982, Astrophys.Sp.Sci., 86, 117.

Efremov, Yu.N. and Pavlovskaya, E.D. 1982, Pis 'ma Astron.Zh., 8, 9 (Sov.Astron.Letts. 8, 4).

Eggen, O.J. 1965, in Stars and Stellar Systems, Galactic Structure, 5, ed. A. Blaauw and M. Schmidt, (Chicago: University of Chicago Press), p. 111.

Elmegreen, B.G. 1979, Astrophys.J., 231, 372.

Elmegreen, B.G. 1982a, Astrophys.J., 253, 655.

Elmegreen, B.G. 1982b, in Sub-Millimeter Wave Astronomy, ed. J.E. Beckman and J.P. Phillips, (Cambridge: Cambridge Univ. Press), p. 1.

Elmegreen, B.G. 1983, Monthly Not.Roy.Astron.Soc., 203, 1011.

Elmegreen, B.G. 1985a, in Birth and Evolution of Massive Stars and Stellar Groups, ed. W. Boland and H. van Woerden, (Dordrecht: Reidel), p. 227.

Elmegreen, B.G. 1985b, in Birth and Infancy of Stars, ed. R. Lucas, A. Omont and R. Stora, (Amsterdam: Elsevier Science Publishers B.V.), p. 215.

Elmegreen, B.G. and Lada, C.J. 1977, Astrophys.J., 214, 725.

Elmegreen, B.G. and Elmegreen, D.M. 1983a, Astrophys.J., $267,31$.

Elmegreen, B.G. and Elmegreen, D.M. 1983b, Monthly Not.Roy.Astron.Soc., 203 , 31.

Elmegreen, B.G. and Elmegreen, D.M. 1985, Astrophys.J., 288, 438.

Elmegreen, B.G. and Elmegreen, D.M. 1986, in preparation, (EE86).

Elmegreen, D.M. 1980, Astrophys.J.Suppl., 43, 37.

Elmegreen, D.M. 1981, Astrophys.J.Suppl., 47, 229.

Elmegreen, D.M. and Elmegreen, B.G. 1982, Monthly Not.Roy.Astron.Soc., 201 , 1021.

Elmegreen, D.M. and Elmegreen, B.G. 1984, Astrophys.J.Suppl., 54 , 127.

Elmegreen, D.M. and Elmegreen, B.G. 1986, in preparation.

Emerson, D.T. 1974, Monthly Not.Roy.Astron.Soc., 169, 607.

Feitzinger, J.V. and Schwerdtfeger, H. 1982, Astron.Astrophys., $116,117$.

Feitzinger, J.V. and Seiden, P.E. 1983, in Internal Kinematics and Dynamics of Galaxies,

IAU Symposium No. 100, ed. E. Athanassoula, (Dordrecht: Reidel), p. 137.

Franco, J. and Shore, S.N. 1984, Astrophys.J., 285, 813. 
Freedman, W.L. and Madore, B.F. 1984, Astrophys.J., 280, 592.

Freeman, K.C. 1970, Astrophys.J., 160, 811.

Fukunaga, M. and Tosa, M. 1987, in Star Forming Regions, IAU Symposium No. 115, ed. M. Peimbert and J. Jugaku, (Dordrecht: Reidel).

Gallagher, J.S. and Hunter, D.A. 1983, Astrophys.J., 274, 141.

Gallagher, J.S. and Hunter, D.A. 1984, Ann.Rev.Astron.Astrophys., 22, 37.

Gehrz, R.D., Sramek, R.A. and Weedman, D.W. 1983, Astrophys.J., 267 , 551.

Geller, M. and Huchra, J. 1983, Astrophys.J.Suppl., 52, 61.

Georgelin, Y.M. and Georgelin, Y.P. 1976, Astron.Astrophys., 49, 57.

Gerola, H. and Seiden, P.E. 1978, Astrophys.J., 223, 129.

Gerola, H. and Seiden, P.E. 1979, in Photometry, Kinematics and Dynamics of Galaxies, ed. D.S. Evans, (Austin: The University of Texas Press), p. 475.

Gerola, H., Seiden, P.E. and Schulman, L.S. 1980, Astrophys.J., 242, 517.

Goldreich, P. and Lynden-Bell, D. 1965, Monthly Not.Roy.Astron.Soc., 130 , 97.

Gosachinskij, I.V. and Khersonskij, V.K. 1982, Astron.Zh., 59, 237 (Sov.Astron., 26,146).

Grabelsky, D.A. 1985, Ph.D. Dissertation, Columbia University.

Grosb $\varnothing 1$, P.J. 1977, Astron.Astrophys., 57, 461.

Gum, C.S. and de Vaucouleurs, G. 1953, Observatory, 73, 152.

Güsten, R. and Mezger, P.G. 1983, Vistas Astron., 26, 159.

Hamajima, K. and Tosa, M. 1975, Pub.Astron.Soc.Japan, 27, 561.

Hausman, M.A. and Roberts, W.W., Jr. 1984, Astrophys.J., 282, 106.

Hayward, R. 1964, Pub.Astron.Soc.Pacific, 76, 35.

Heidmann, J. 1983, Highlights of Astronomy, 6, 609.

Heiles, C. 1979, Astrophys.J., 229, 533.

Henderson, A.P., Jackson, P.D. and Kerr, F.J. 1982, Astrophys.J., 263 , 116.

Herbst, W. and Assousa, G.E. 1977, Astrophys.J., $217,473$.

Hilton, J.L. and Bash, F. 1982, Astrophys.J., 255, 217.

Hindman, J.V. 1967, Aust.J.Phys., 20, 147.

Hippelein, H. and Fried, J.W. 1984, Astron.Astrophys., 141, 49.

Hodge, P.W. 1967, Pub.Astron.Soc.Pacific, 79, 29.

Hubble, E.P. 1936, Astrophys.J., 84, 158.

Huchra, J. and Geller, M. 1982, Astrophys.J., 257, 423.

Hughes, V.A. and Routledge, D. 1972, Astron.J., 77, 210.

Humason, M.L., Mayall, N.V. and Sandage, A.R. 1956, Astron.J., 61 , 97.

Humphreys, R.M. and Sandage, A. 1980, Astrophys.J.Suppl., 44, 319.

Hunter, D. 1982, Astrophys.J., 260, 81.

Hunter, J.H., Jr. and Horak, T. 1983, Astrophys.J., 265, 402.

Ichikawa, T., Nakano, M., Tanaka, Y.D., Saito, M., Nakai, N., Sofue, Y. and Kaifu, N. 1985, Pub.Astron.Soc.Japan, 37, 439.

Ichikawa, T., Nakano, M. and Tanaka, Y.D. 1987, in IAU Sympoisium No. 115, Star

Forming Regions, ed. M. Peimbert and J. Jugaku, (Dordrecht: Reidel).

Ikeuchi, S., Habe, A. and Tanaka, K. 1984, Monthly Not.Roy.Astron.Soc., 207 , 909.

Israel, F. 1980, Astron.Astrophys., 90, 246.

Israel, F.P., Goss, W.M. and Allen, R.J. 1975, Astron.Astrophys., 40 , 421.

Jensen, E.B. 1977, Ph.D. Dissertation, University of Arizona.

Jensen, E.B., Strom, K. and Strom, S.E. 1976, Astrophys.J., 209, 748.

Jog, C. and Solomon, P.M. 1984, Astrophys.J., 276, 114.

Jura, M. 1976, Astron.J., 81, 178.

Kalnajs, A.J. 1972, Astrophys.Letts., 11, 41.

Kalnajs, A.J. 1978, in IAU Symposium No. 77, Structure and Properties of Nearby Galaxies, ed. E.M. Berkhuijsen and R. Weilebinski, (Dordrecht: Reidel), p. 113. 
Kaufman M. 1981, Astrophys.J., 250, 534.

Kennicutt, R.C. 1983, Astrophys.J., 272, 54.

Kennicutt, R.C. 1984, Astrophys.J., 287, 116.

Kennicutt, R.C. and Hodge, P.W. 1980, Astrophys.J., 241, 573.

Kennicutt, R.C. and Hodge, P.W. 1982, Astrophys.J., 253, 101.

Kennicutt, R.C. and Hodge, P.W. 1984, Pub.Astron.Soc.Pac., 96, 944.

Kennicutt, R.C. and Edgar, B.K. 1986, Astrophys.J., 300, in press.

Kevanishvili, G. Th. 1969, Byull.Abastumansk.Astrofiz.Obs. , No. 37, 117.

Kevanishvili, G. Th. 1975, Byull.Abastumansk.Astrofiz.Obs., No. 46, 101.

Khachikian, E.Ye. 1984, Astronomy with Schmidt-type Telescopes, ed. M. Capaccioli,

(Dordrecht: Reidel), p. 427.

Kulkarni, S.R. 1983, Ph.D. Dissertation, University of California at Berkeley.

Kwan, J. and Valdes, F. 1983, Astrophys.J., 271, 604.

Lacey, C.G. and Fall, S.M. 1985, Astrophys.J., 290, 154.

Larson, R.B. 1980, Phil.Trans.Roy.Soc.London, Ser.A, 269, 299.

Larson, R.B. 1982, Monthly Not.Roy.Astron.Soc., 200, 159.

Lattanzio, J.L., Monaghan, J.J., Pongracic, H. and Schwarz, M.P. 1985, Monthly Not.Roy.Astron.Soc., 215, 125.

Latyshev, I.N. 1980, Astron.Tsirk., 1141, 7.

Lesh, J.R. 1968, Astrophys.J.Suppl., 17, 371.

Liebovitch, L.S. and Lin, C.C. 1978, Bull.American Astron.Soc., 9 , 639.

Lin, C.C. and Shu, F.H. 1964, Astrophys.J., 140, 646.

Lin, C.C. and Shu, F.H. 1967, in Radio Astronomy and the Galactic System, IAU Symposium No. 31, ed. H. van Woerden, (London: Academic Press), p. 313.

Lin, C.C. 1968, in Galaxies and the Universe, ed. L. Woltjer, (New York: Columbia University Press), p. 33.

Lindblad, P.O. 1967, Bull.Astron.Inst.Neth., 19, 34.

Lindblad, P.O., Grope, K., Sandqvist, Aa. and Schober, J. 1973, Astron.Astrophys., $24,309$.

Linke, R.A. 1982, in Extragalactic Molecules, ed. L. Blitz and M. Kutner, (Green Bank: NRAO Publications Office), p. 87.

Loose, H.H. and Fricke, K. 1982a, Mitt.Astron.Ges., 55, 100.

Loose, H.H. and Fricke, K. 1982b, in The Most Massive Stars, ESO Workshop, Garching, p. 269.

Loose, H.H., Krügel, E. and Tutukov, A. 1982, Astron.Astrophys., 105, 342.

Lortet, M.C., Testor, G., Heydari-Malayeri, M. and Niemela, V. 1987, in Star Forming Regions, IAU Symposium No. 115, ed. M. Peimbert and J. Jugaku, (Dordrecht: Reidel).

Lynden-Bell, D. 1966, Observatory, 86, 57.

Lyng̊, G. and Wramdemark, S. 1984, Astron.Astrophys., 132, 58.

Mathewson, D.S., van der Kruit, P.C. and Brouw, W.N. 1972, Astron.Astrophys., 17 , 468.

McCall, M.L. 1982, Ph.D. Dissertation, University of Texas, Publ. in Astronomy No. 20.

McGee, R.X. and Milton, J.A. 1964, Austral.J.Phys., 17, 128.

McGee, R.X. and Milton, J.A. 1966, Austral.J.Phys., 19, 343.

Meaburn, J. 1980, Monthly Not.Roy.Astron.Soc., 192, 365.

Melnick, J., Moles, M. and Terlevich, R. 1985, Astron.Astrophys., 149, L24.

Mezger, P.G. 1970, in The Spiral Structure of the Galaxy, IAU Symposium No. 38, ed. W.

Becker and G. Contopoulos, (Dordrecht: Reidel), p. 107.

Moffat, A.F.J., Seggewiss, W. and Shara, M.M. 1985, Astrophys.J., 295, 109.

Moore, J.H. and FitzGerald, P.M. 1973, J.Roy.Astron.Soc.Canada, 67 , 291.

Morgan, W.W., Sharpless, S. and Osterbrock, D. 1952, Astron.J., 57, 3.

Morgan, W.W., Whitford, A.E. and Code, A.D. 1953, Astrophys.J., 118, 318. 
Morozov, A.G. 1985, Astron.Zh., 62 , 209 (Soviet Astron., 29, 120).

Mountain, C.M., Hawarden, T.G., Joseph, R.D., Leggett, S.K. 1987, in Star Forming Regions, IAU Symposium No. 115, ed. M. Peimbert and J. Jugaku, (Dordrecht: Reidel).

Mouschovias, T. Ch., Shu, F.H. and Woodward, P. 1974, Astron.Astrophys., 33 , 73.

Mueller, M.W. and Arnett, W.D. 1976, Astrophys.J., $210,670$.

Myers, P.C., Dame, T.M., Thaddeus, P., Cohen, R.S., Silverberg, R.F., Dwek, E. and Hauser, M.G. 1986, Astrophys.J., 301, in press.

Nepveu, M. and Robnik, M. 1984, Astron.Astrophys., 138, 201.

Newton, K. 1980a, Monthly Not.Roy.Astron.Soc., 190, 689.

Newton, K. 1980b, Monthly Not.Roy.Astron.Soc., 191, 615.

Nozakura, T. and Ikeuchi, S. 1984, Astrophys.J., 279, 40.

Ögelman, H.B. and Maran, S.P. 1976, Astrophys.J., 209, 124.

Olano, C.A. 1982, Astron.Astrophys., $112,195$.

Ondrechen, M.P. 1985, Astron.J., 90, 1474.

Oort, J.H. 1954, Bull.Astron.Inst.Neth., 12, 177.

Oort, J.H. 1962, in Interstellar Matter in Galaxies, ed. L. Woltjer, (New York: Benjamin), p. 234.

Öpik, E.J. 1953, Irish Astron.J., 2, 219.

Page, T. and Carruthers, G.R. 1981, Astrophys.J., 248, 906.

Pagel, B.E.J. and Edmunds, M.G. 1981, Ann.Rev.Astron.Astrophys., 19, 77.

Pellet, A., Astier, N., Viale, A., Courtès, G., Maucherat, A., Monnet, G., Simien, F. 1978, Astron.Astrophys.Suppl., 31, 439.

Pérault, M., Falgarone, E. and Puget, J.L. 1985, Astron.Astrophys., 152 , 371.

Persi, P., Tapia, M., Roth, M. and Ferrari-Toniolo, M. 1985, Astron.Astrophys., 144, 275.

Petrosyan, A.R., Saakyan, K.A., Khachikyan, Eh.E. 1984a, Astrofizika, 19, 619 (Astrophys., 19, No. 4).

Petrosyan, A.R., Saakyan, K.A., Khachikyan, Eh.E. 1984b, Astrofizika, 20, 51 (Astrophys., 20, No. 1).

Petrosyan, A.R., Saakyan, K.A., Khachikyan, Eh.E. 1984c, Astrofizika, 21, 57 (Astrophys., 21, No. 1).

Phillips, S. and Disney, M. 1985, preprint.

Pikel'ner, S.B. 1970, Astron.Zh.Akad.Nauk SSSR, 47 , 752 (Sov.Astron.AJ 14, No.4).

Pismis, P. and Hasse, I. 1982, Rev.Mex.Astron.Astrophys., 5, 161.

Quiroga, R.J. and Schlosser, W. 1977, Astron.Astrophys., 57, 455.

Roberts, W.W., Jr. 1969, Astrophys.J., 158, 123.

Roberts, W.W., Jr. and Yuan, C. 1970, Astrophys.J., 161, 877.

Roberts, W.W., Jr. and Shu, F.H. 1972, Astrophys.Letts., 12, 43.

Roberts, W.W., Jr., Roberts, M.S. and Shu, F.H. 1975, Astrophys.J., $196,381$.

Roberts, W.W., Jr. and Hausman, M.A. 1984, Astrophys.J., 277, 744.

Romanishin, W. 1985, Astrophys.J., 289, 570.

Rosa, M. and Solf, J. 1984, Astron.Astrophys., 130, 29.

Rosa, M., Joubert, M. and Benvenuti, P. 1984, Astron.Astrophys.Suppl., 57, 361.

Rots, A.H. 1975, Astron.Astrophys., 45, 43.

Rumstay, K.S. and Kaufman, M. 1983, Astrophys.J., 274, 611.

Safronov, V.S. 1960, Ann.d'Astrophys., 23, 979.

Sandage, A. 1961, The Hubble Atlas of Galaxies, (Washington: The Carnegie Institute of Washington).

Sandage, A. and Tammann, G.A. 1981, A Revised Shapley Ames Catalog of Galaxies,

(Washington: Carnegie Institute of Washington).

Sandage, A., Binggeli, B. and Tammann, G.A. 1985, Astron.J., 90 , 395. 
Sanders, R.H. and Huntley, J.M. 1976, Astrophys.J., 209, 53.

Sanders, R.H. and Tubbs, A.D. 1980, Astrophys.J., 235, 803.

Sanz Fernández de Córdoba, L. and Benvenuti, P. 1984, ESA Special Pub.ESA SP-218, p. 73.

Sargent, W.L.W. and Searle, L. 1970, Astrophys.J.(Letts.), 162 , L155.

Schlosser, W. and Görnandt, V. 1984, Astron.Astrophys., 137, 287.

Schlosser, W. and Musculus, D. 1984, Astron.Astrophys., 131, 367.

Schmidt, M. 1959, Astrophys.J., 129, 243.

Schmidt-Kaler, Th. and Schlosser, W. 1973, Astron.Astrophys., 25, 191.

Schweizer, F. 1976, Astrophys.J.Suppl., 31, 313.

Scoville, N. and Young, J.S. 1983, Astrophys.J., 265, 148.

Seiden, P.E. 1983, Astrophys.J., 266, 555.

Seiden, P.E. and Gerola, H. 1979, Astrophys.J., 233, 56.

Seiden, P.E., Schulman, L.S. and Gerola, H. 1979, Astrophys.J., $232,702$.

Seiden, P.E. and Gerola, H. 1982, Fundamentals of Cosmic Physics, 7, 241.

Seiden, P.E., Schulman. L.S. and Feitzinger, J.V. 1982, Astrophys.J., 253 , 91.

Seiden, P.E., Schulman, L.S. and Elmegreen, B.G. 1984, Astrophys.J., 282, 95.

Sellwood, J.A. and Carlberg, R.G. 1984, Astrophys.J., 282, 61.

Shahbazian, R.K. 1968, Astrofizika, 4, 273.

Shahbazian, R.K. 1970, Astrofizika, 6, 367.

Shevchenko, V.S. 1979, Astron.Zh., 56, 279 (Sov.Astron.AJ. 23, 163).

Shevchenko, V.S. 1980, Astron.Zh., 57, 1162 (Sov.Astron.AJ. 24, 670).

Shore, S.N. 1981, Astrophys.J., 249, 93.

Shore, S.N. 1983, Astrophys.J., 265, 202.

Shu, F.H., Stachnik, R.V. and Yost, J.C. 1971, Astrophys.J., 166 , 465.

Shu, F.H., Milione, V., Gebel, W., Yuan, C., Goldsmith, D.W. and Roberts, W.W., Jr. 1972, Astrophys.J., 173, 557.

Skillman, E.D. 1985, Astrophys.J., 290, 449.

Skillman, E.D. and Balick, B. 1984, Astrophys.J., 280, 580.

Smith, G., Elmegreen, B.G. and Elmegreen, D.M. 1984, Monthly Not.Roy.Astron.Soc., 210 , 399.

Solomon, P. 1983, in Internal Kinematics and Dynamics of Galaxies, IAU Symposium No. 100, ed. E. Athanassoula, (Dordrecht: Reidel), p. 35.

Solomon, P.M. and Sanders, D.B. 1980, in Giant Molecular Clouds in the Galaxy, eds. P.M. Solomon and M.G. Edmunds, (New York: Pergamon), p. 41.

Solomon, P., Sanders, D.B. and Rivolo, A.R. 1984, Astrophys.J.(Letts.), 292, L19.

Stal'bovskii, O.I. and Shevchenko, V.S. 1981, Astron.Zh., 58 , 45 (Sov.Astron.AJ. 25, 25).

Stark, A.A. 1979, Ph.D. Dissertation, Princeton University.

Stark, A.A. 1985, in The Milky Way Galaxy, IAU Symp. No. 106, ed. H. van Woerden, R.J. Allen and W.B. Burton, (Dordrecht: Reidel), p. 445.

Stark, A.A. and Blitz, L. 1978, Astrophys.J.(Letts.), 225, L15.

Stone, M.E. 1970, Astrophys.J., 159, 293.

Stothers, R. and Frogel, J.A. 1974, Astron.J., 79, 456.

Strom, S.E. 1980, Astrophys.J., 237, 686.

Strom, S., Jensen, E. and Strom, K. 1976, Astrophys.J.(Letts.), 206, L11.

Struck-Marcell, C. and Scalo, J.M. 1984, Astrophys.J., 277, 132.

Talbot, R.J., Jr. 1980, Astrophys.J., 235, 821.

Talbot, R.J., Jr. and Arnett, W.D. 1975, Astrophys.J., 197, 551.

Talbot, R.J., Jr., Jensen, E.B. and Dufour, R.J. 1979, Astrophys.J., 229 , 91.

Telesco, C.M. 1984, in Occasional Reports Roy. Obs. Edinburgh, Proceedings of the Workshop on Star Formation, ed. R.D. Wolstencroft, No. 13. 
Terlevich, R. 1984, Observatory, 104, 59.

Terlevich, R. and Melnick, J. 1981, Monthly Not.Roy.Astron.Soc., 195 , 839.

Tomisaka, K. 1984, Publ.Astron.Soc.Japan, 36, 457.

Tomisaka, K. 1985, preprint.

Tomisaka, K., Habe, A. and Ikeuchi, S. 1981, Astrophys.Sp.Sci., 78 , 273.

Toomre, A. 1964, Astrophys.J., 139, 1217.

Toomre, A. 1981, in The Structure and Evolution of Normal Galaxies, ed. S.M. Fall and D. Lynden-Bell, (Cambridge: Cambridge Univ. Press), p. 111.

Turner, E.L. 1976, Astrophys.J., 208, 20.

Turner, E.L. and Gott, J.R. 1976, Astrophys.J.Suppl., 32, 409.

Unwin, S.C. 1980a, Monthly Not.Roy.Astron.Soc., 190, 551.

Unwin, S.C. 1980b, Monthly Not.Roy.Astron.Soc., 192, 243.

van Albada, G.D. 1980, Astron.Astrophys., 90, 123.

van den Bergh, S. 1960, Astrophys.J., 131, 215.

Venger, A.P., Gosachinskij, I.V., Grachev, V.G., Egorova, T.M., Ryzhkov, N.F. and

Khersonskij, V.K. 1984, Astrophys.Sp.Sci., 107, 271.

Viallefond, F., Allen, R.J. and Goss, W.M. 1981, Astron.Astrophys., $104,127$.

Viallefond, F., Goss, W.M. and Allen, R.J. 1982, Astron.Astrophys., 115 , 373.

Viallefond, F., Donas, J. and Goss, W.M. 1983, Astron.Astrophys., 119 , 185.

Wakamatsu, K. and Nishida, M.T. 1980, Pub.Astron.Soc.Japan, 32, 389.

Weaver, H. 1979, in Large Scale Characteristics of the Galaxy, IAU Symposium No. 84, ed. W.B. Burton, (Dordrecht: Reidel), p. 295.

Weedman, D.W., Feldman, F.R., Balzano, V.A., Ramsey, L.W., Sramek, R.A. and Wu, C.C. 1981, Astrophys.J., 248, 105.

Westerlund, B.E. and Mathewson, D.S. 1966, Monthly Not.Roy.Astron.Soc., 131 , 371.

Wevers, B.M.H.R. 1984, Ph.D. Dissertation, Groningen.

Woltjer, L. 1965, in Stars and Stellar Systems, Galactic Structure, 5, ed. A. Blaauw and M.

Schmidt, (Chicago: University of Chicago Press), p. 531.

Woodward, P.R. 1976, Astrophys.J., 207, 484.

Wouterloot, J. 1981, Ph.D. Dissertation, Leiden.

Wray, J.D. and de Vaucouleurs, G. 1980, Astron.J., 85, 1.

Wright, M.C.H. 1971, Astrophys.J., 166, 455.

Wynn-Williams, C.G. and Becklin, E.E. 1985, Astrophys.J., 290, 108.

Young, J.S. and Scoville, N. 1982a, Astrophys.J., 258, 467.

Young, J.S. and Scoville, N. 1982b, Astrophys.J.(Letts.), 260 , L11.

Yuan, C. and Grosbøl, P.J. 1981, Astrophys.J., 243, 432.

YOUNG: Kennicutt's H $\alpha$ equivalent widths apply to galaxies as a whole, not arm vs. interarm regions within a galaxy. In order to verify that the star formation rate per unit $\mathrm{H}_{2}$ mass is constant in grand design arms vs. flocculent arms one needs to observe galaxies in detail to measure the $\mathrm{H \alpha} / \mathrm{CO}$ ratio and $\mathrm{H}_{2} / \mathrm{HI}$ ratios in arm and interarm regions in galaxies of both types.

ELMEGREEN: Perhaps the most important point of my discussion is that we should not accept the classical triggering hypothesis without first obtaining careful and unambiguous observations such as those you suggest, which isolate the possible triggering effect from all of the other subtle aspects of gas flow in density waves.

STROM: I think you have performed a valuable service in reminding us 
of two points: the underlying density waves of ten have large amplitudes (and bring attention to the star-forming regions) and that large molecular clouds form near the density wave crests. Your major conclusions, however, rest on two points: 1) the lack of "blue arms" associated with density wave crests and (2) the lack of separation between "flocculent" and "grand design" spirals as a function of Hubble or arm type. I think that the first result is in part explained by the relative insensivity of $(B-V)$ to star formation. Studies by Schweizer and later by Jensen, Talbot, and Dufour (for M83) show clear evidence in U-B for "blueing" near the arm crests. Correct care was given in the later paper to subtract out the spiral arm contribution. Perhaps a careful study of star forming yields based on $U-B$ or $\mathrm{H} \alpha$ fluxes (rather than counts of HII regions) would be revealing. With regard to the second point, did you search for a luminosity dependence of SFR at a fixed Hubble type when you compared the flocculent to grand design systems? In particular, is there any tendency for the floccular galaxies to be somewhat more luminous at a fixed Hubble type?

ELMEGREEN: I believe that spiral arms are bluer than the interarm regions, but only because the arm/interarm contrast in the gas exceeds the arm/interarm contrast in the old stars not because of triggered star formation. If star formation merely follows the gas, and occurs at a rate per unit gas mass that is independent of phase in a density wave, then this excess gas contrast alone produces an excess star formation contrast relative to the old stars. Regarding the second point. I did not sort galaxies according to luminosity. I will have to try this.

DOPITA: I strongly agree with your theses. The LMC contains excellent examples of both your superclouds and of propagating star formation (e.g. Constellation III). I would like to emphasize that recent work by Mathewson, Ford, and myself (Astrophys. J. 1985, November) shows the region of star formation propagates outward at constant speed, suggesting both that the overpressure is constant in this region and that it is directly proportional to the local SFR. The gas ejected from the plane in these regions has large velocities ( $\left.35 \mathrm{~km} \mathrm{~s} \mathrm{~s}^{-1}\right)$, and the circulation up to the halo and back has probably a great effect on the HI disk and in controlling the global SFR.

ALLEN: I think no one doubts that star formation is ubiquitous in galaxies. Even the distribution of massive, recently-formed stars is not well ordered in many galaxies as we have know for roughly a decade now since Hodge presented his atlas of HII regions in galaxies. But I want to caution against throwing the baby out with the bath water; density waves can be useful diagnostic tools to study the time sequence of star formation. In many galaxies which have been observed with sufficient linear resolution, we do see long, narrow structures in HI, HII, and dust, with the dust lanes often clearly separated from the H II "string-of-pear1s". Such features in M83 have been recently discussed in detail (Allen, Atherton, and Tilamis; Nature, in press), and it appears that the density wave has indeed started, and thereafter conveniently spread the star formation process out into space across the spiral arms. You seem to be denying this scenario. Then what, in your view, is going on? 
ELMEGREEN: I do not deny the observations you mention, only the inference that the spatial sequence is an evolutionary sequence, that the gas currently making the HII regions was triggered to do so by the dust lanes, or even that this star-forming gas was ever once part of the dust lane. Another possibility is that the interstellar gas contains several components, some of which (the diffuse clouds and intercloud medium) are at low pressure and are dynamically interconnected (by magnetic fields, for example, or by rapid collisions). This component could shock in a density wave producing a dust lane but have nothing to do with star formation. Another component (the dense, self-gravitating molecular clouds) could be at higher pressure and could move more ballistically than diffuse clouds and therefore not shock in the same way in a density wave. This second component could collect and coagulate inside a spiral arm, well beyond the shock in the tenuous component, as shown by recent n-body calculations. Then the dense star-forming component would appear "down stream" from and parallel to, the dust lane, but not be causally connected to it. The observations you mention are suspicious anyway. The dust lane is a local peak in density, and the ridge of $\mathrm{H}$ II regions must be a density peak also, because of the associated molecular clouds. So why does the gas response to a density wave have two peaks, if the gas responds uniformly? How can the gas in the dust lane evolve into the gas in the molecular clouds, downstream, when there is a clear gap in matter separating the two suposedly evolutionary states? I would have expected an intermediate state in this gap, a state between the trigger and the reaction, such as intermediate-density material with no star formation, but the extinction from the dust that should be in this hypothetical state cannot be seen. So the gas response is probably double peaked because the gas is multicomponent. 